\title{
Structural Health Monitoring Applications in Tall Buildings
}

\author{
Yifan Zhao \\ Southwest Jiaotong university Leeds joint school, Sichuan Chengdu 611700 China
}

\begin{abstract}
Since there is not much research on structural health monitoring (SHM) applications in tall buildings nowadays, this paper gives a proposal of how it can be applied on skyscrapers. Covering the whole process of SHM, this paper focuses more on the diagnostic algorithms, including Structural dynamic index method, Modal parameter identification method Neural network algorithm and Genetic algorithm and how these algorithms can be used in SHM. After introducing the basic process of SHM, an example is given to show how these principles can be applied in this over 400m building. And after all these introductions, a conclusion can be drawn that the structural health monitoring system can be applied properly in tall buildings following the way proposed in this paper.
\end{abstract}

\section{Introduction}

First applied in aeronautical industry, the structural health monitoring (SHM) technology is now widely developed in civil engineering, especially in bridges. Some successful applications of structural health monitoring have already been carried out in bridges like the Tsing Ma Bridge in Hong Kong[1]. However, there are only few researches about tall buildings in structural health monitoring area which is worth concerning. Since there are more and more skyscrapers in the world such as Burj Khalifa and Shanghai tower, it is of great significance to figure out how can the structural health monitoring be applied in these tall buildings. The objective of this paper is to learn from the successful SHM applications in bridges and other areas to find out how SHM can be applied in tall buildings. In this paper, the whole process of the SHM is discussed briefly, including the sensors, transmitting process and diagnostic algorithm. Among these, the diagnostic algorithm is of the most concern. Thus, this part is demonstrated in detail. After that, the Chengdu Greenland Center is chosen as the example case and an initial plan for it is proposed.

\section{Brief historical overview}

As the very first application of SHM in civil engineering area, bridges have various of successful cases and have accumulated lots of precious experience such as the Tsing Ma Bridge in Hong Kong, the Seohae Bridge in Korea. Since recently there are more and more large-scale bridges, some useful SHM system have been applied to make up the monitoring system in the over $1000 \mathrm{~m}$ bridges such as, the Tsing Ma Bridge with a length of $1300 \mathrm{~m}$, Shanghai Chongming Crossing with a length of $1200 \mathrm{~m}$ and the Messina Strait Bridge with a length of $3000 \mathrm{~m}[1]$.
Nowadays, people start to focus on the SHM application in tall buildings since there are more and more skyscrapers in the world. And some of them have already installed the SHM successfully like the Ping-An Finance Center in China which carried 553 sensors in total[1]. However, this area is still in lack of study.

\section{Sensing system}

The first part of structural health monitoring is the sensing system that involves the data collection, data transmission and process[2]. In order to get the information of the building, it is of vital significance to get the features using all kinds of sensors and transmit them to the process center to identify the damage.

\subsection{Sensor}

As the development of material and technology, the materials of sensors used silica, ceramic material and so on. Among these, the optical fibre and wireless sensor stands out in recent years after lessons learned from years of practice[1]. These two sorts of sensor are restricted by the immature of technology and the economic issues which are handled now. As for the optical fibre sensors, several bridges have already installed and used them to form the sensing system which can detect parameters like strain, temperature, chemical owing to its good stability and durability. The wireless sensors can be installed in any place so that they are more suitable to be applied in tall buildings. However, the stability of them is not stratifying and the security of the sensor network is of great concern. The former problem will be discussed in detail in the next section. Other sensors including Ferroelectric sensor which detects cracks and fatigue, MEMS sensor which detects vibration and force, Pyroelectric that measures the temperature and so on[3] are also used in the sensing system. 


\subsection{Data transmission}

Once the data is collected by the sensors, it is of vital importance to transmit and process the data. However, the wireless sensor lacks stability compared to the traditional sensor where the data may be lost during the transmission. In order to solve this problem, Professor Hui Li proposed a recovery method that is based on compressive sensing theory which process the data using Doubly stochastic matrix before data transmission[4]. By doing that, even the data is lost during the transmission process, they can be recovered afterwards. According to Hui $\mathrm{Li}$ and her team's test, this algorithm performs well with a $10 \%$ loss of data which is pretty satisfying[2].

\section{Damage identification}

The identification process can be divided into 2 parts: first is to decide which factors have more influence on the tall buildings and how to detect them. Next is to diagnose damage using the diagnostic algorithms.

\subsection{Impact factor}

\subsubsection{Wind load}

In fact, the wind load is one of the top concerns of tall buildings because of the high wind speed, the long natural period and low structural damping. Thus, the wind load in super-tall buildings needs to be taken into consideration carefully. Take the building as a cantilever, the axil force $\mathrm{N}$, moment $\mathrm{M}$ and lateral displacement $\mathrm{s}$ can be represented by the high $\mathrm{H}$ :

$$
\begin{gathered}
N=W H \propto H \\
M=\frac{q H^{2}}{2} \propto H^{2} \\
S=\frac{q H^{4}}{8 E I} \propto H^{4}
\end{gathered}
$$

Thus, it can be concluded that the tall buildings whose height $\mathrm{H}$ are very large should pay more attention on lateral load, especially the wind load.

\subsubsection{Structural vibration}

The structure will vibrate under external load and once the load exceeds the boundary that will damage the structure. However, the external load may not lead to notable changes in strain or visible cracks in the building while the structure has been damaged. In order to detect the damage, the natural frequency and mode of the vibration with lower threshold value to load are used. Thus, detecting the vibration of the structure instead of strain and cracks can deduce the load condition more precisely.

\subsubsection{Temperature \& Humidity}

During the data collecting process, the change of environment like temperature and humidity can influence the final results of the data. Hence, it is important to test the environment change at the same time and eliminate these noises by algorithms. Take the temperature for example, it is able to affect the modal frequency by $10 \%$ which can influence structural damage detection easily[1]. After collecting enough data of temperature and modal frequency, the effects of environment and structural damage can be distinguished using neural network or support vector machine which will be discussed thoroughly in 4.2 .3 neural network.

\subsection{Diagnostic algorithm}

\subsubsection{Structural dynamic index method}

With the help of extreme large number of data, each damage status can correspond to one unique structural dynamic index and that is how this system works. For one specific dynamic feature like the vibration frequency, the correlation between them can be derived directly. However, this will not work when the features become larger and that is true in real situations. Thus, a database should be built for this scenario.

\subsubsection{Modal parameter identification method}

Frequency-domin method is to transform the data collected in time-domin to frequencies using Fourier transform and correlate the frequencies to the modal parameter obtained in the database. Derived from the structural dynamic equation, the equation of motion $\mathrm{f}$ is as follows:

$$
f=\left(-\omega^{2} M+j \omega C+K\right) y
$$

And the Frequency response function can be obtained as follows:

$$
H(\omega)=\left(-\omega^{2} M+j \omega C+K\right)^{-1}
$$

where the Harmonic displacement vector $y=H(\omega) f$. Assuming harmonic response, the solution can be represented by:

$$
H(\omega)=\sum_{i=1}^{N} \frac{\emptyset_{i} \emptyset_{i}{ }^{T}}{\left(\omega_{i}^{2}-\omega^{2}+2 j \zeta \omega_{i} \omega\right)}
$$

Solving the frequency response function also depends on the number of Degree of Freedom (DOF). For both single-DOF and multi-DOF problem, the theoretical Frequency response function needs to fit with the measured Frequency response function[5]. The advantage of time-domin method is that it can avoid structural component resonance with external load and the calculation work is much smaller than the time-domin method.

With the development of computer technology, the time-related parameter such as accelerate, velocity can be used directly by time-domin identification algorithm. Typical methods include polyreference complex exponential (PRCE), Ibrahim time domain (ITD), eigensystem realization algorithm (ERA)[6]. The problem is solved by calculating the Impulse response function which is also the inverse Fourier transforms of the Frequency response function: 


$$
\begin{aligned}
& f(\omega)=\int_{-\infty}^{\infty} F(t) e^{2 \pi i t \omega} d t \\
& F(t)=\int_{-\infty}^{\infty} f(\omega) e^{2 \pi i t \omega} d \omega
\end{aligned}
$$

The time-domin method does not involve the transformation of the original data, avoiding the errors and information lost in this process. However, the time-domin method is affected by the noise and computational parameter greatly because of the heavy calculation process.

\subsubsection{Neural network algorithm}

In order to classify the damages, the neural network algorithm is widely used since it can solve the complex non-linear relationship problem easily. Typically, the neural network is composed by 3 parts: input layer, hidden layer and output layer[7].

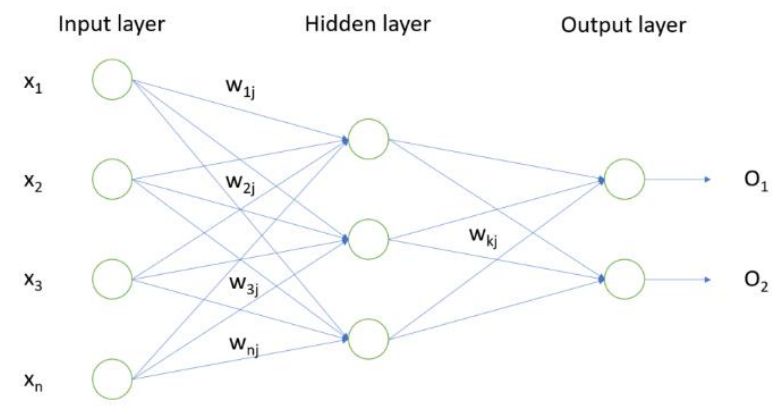

Fig. 1. Neural network

The values given in input layer $\mathrm{x} 1-\mathrm{xn}$ is multiplied by their weight $w \mathrm{j}, \mathrm{k}$ to form the input in the hidden layer $\mathrm{zn}$ like it is shown in Equation 1-1[8]:

$$
z=g\left(x_{1} \times w_{1,1}+x_{2} \times w_{1,2}+\cdots x_{n} \times w_{1, n}\right)
$$

In order to determine the weight $\mathrm{W}$ in each layer, typically 3 algorithms are used in SHM area, including Back propagation, Radial basis function and Selforganizing neural network. The neural network algorithm has a strong generalization ability that can perform well in the untrained sample. Thus, noise-identification problems mentioned in 4.1.3 can use neural network algorithm to distinguish two features.

For instance, a number of frequency data $\left(\mathrm{x}_{1}, \mathrm{x}_{2}\right.$, $\left.\mathrm{x}_{3} \ldots \ldots \mathrm{x}_{\mathrm{n}}\right)$ can be put into the input layer first after a series of operations such as normalization and regularization. Then these data transmitted to the hidden layer are multiplied by the weight $\mathrm{w}_{\mathrm{j}, \mathrm{k}}$ as shown in equation (4-4). Typically, 2 or 3 hidden layers are used and the original data then can be classified into two categories $\left(\mathrm{O}_{1}, \mathrm{O}_{2}\right)$ : frequencies introduced by the change of environment and frequencies caused by structural damage.

\subsubsection{Genetic algorithm}

Genetic algorithm is a random search method, which simulates the natural evolution. By imitating the process of variation of genes, this algorithm can find the optimal solution quickly. Take a simple genetic algorithm for example: There are $n$ species $\mathrm{Sn}$ at first representing $n$ kinds of possible solutions. And each species has a fitness value $\mathrm{fn}$ which is calculated by a number of evaluation equations that related to the noise-resistance, detection sensitivity and so on. The rank of fitness is represented by $\mu_{\mathrm{n}}$ where the highest fitness has the value of 1 .

\section{Step 1: Selection:}

Like the theory of evaluation, the higher the fitness is, the more possible it will survive. First is to calculate the possibility of each specie by:

$$
P\left(S_{n}\right)=a(1-a)^{\mu_{n}-1}
$$

where $a \in(0,1)$. According to this possibility, the species are selected $n$ times until forming a new group of specie.

\section{Step 2: Crossover:}

Given the new group of species, they are paired to exchange one piece of information to each other which locates on the same position of each specie. This exchanged information is just like the homologous chromosomes synapsis that produces numerous new species. By doing the crossover, the search ability of this algorithm is increased greatly.

\section{Step 3: Mutation:}

At first, the mutation possibility $\mathrm{Pm}$ needs to be allocated to each specie. And according to Pm, part of Sn is selected and mutate to change the original specie. Typical mutation methods include Simple Mutation, Uniform Mutation and Boundary Mutation.

After carrying out these 3 steps, the fitness fn of the specie can reach up to a threshold value, indicating an optimal solution and that is when the algorithm stops.

The property of its quick searching shows its ability can be used to determine the weight parameter mentioned in 4.2.3 Neural network algorithm. The gradient decent method used in neural network algorithm depends highly on the choose of weight. If the weight is inappropriate, the gradient decent may converge slowly or even diverge. Nevertheless, genetic algorithm can help finding the best weight of the layer, accelerating the speed of converge and therefore optimise the algorithm.

\section{Example analysis}

\subsection{Brief introduction}

There is a skyscraper under construction in Chengdu, China, called Chengdu Greenland Center which is going to be the $4^{\text {th }}$ highest building in China and $7^{\text {th }}$ in the world with a total height of $468 \mathrm{~m}$. It has a floor area of over $220000 \mathrm{~m}^{2}$ in total and 101 storeys[9]. For a skyscraper like this, the detections by man can cost a large amount of money so the Chengdu Greenland Center is certainly in need of the help of SHM system to monitor its condition. 


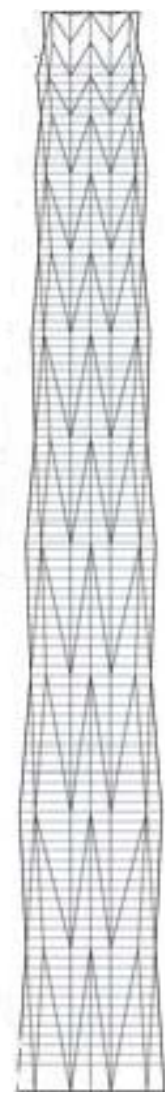

Fig. 2. Sketch of the Chengdu Greenland Center

\subsection{Initial plan for this building}

\subsubsection{Sensing system}

Since this building has a height of $468 \mathrm{~m}$, some traditional sensors like lacer has a poor accuracy during this circumstance. Take lacer for example, it is more likely to be interfered in the up air. Hence, the optical fibre sensors with great accuracy and stability should be widely deployed in this new building to undertake the job such as strain and temperature measurement. Also, as a super-tall building, the anemometers are necessary to detect the wind load, which is the dominant load for this building. As the core of this structure, the Core-Tube takes up the responsibility to resist horizontal forces such as wind load. Thus, the sensors are deployed along the core-tube to detect its condition.

\subsubsection{Damage identification}

In order to determine whether the building is damaged, the structural dynamic index method is used based on the enormous database. In this initial plan, a simple proposal for identification process should be noise-elimination and solving the frequency response function problem.

As it is mentioned above, the interference of environment could be a huge problem in damage identification. Thus, the neural network algorithm with at least 1 2 hidden layers should be used to distinguish the vibrations from structural damage and the environment changes. Also, in order to have a quicker converge speed, the genetic algorithm can be carried out to set an appropriate initial weight matrix.

After eliminating the noises by neural network algorithm, the data like natural frequency $\omega$ are used to establish the Frequency response function. The algorithm is able to determine if this building is damaged after solving the frequency response function. below:

The whole process can be illustrated as the flow chart

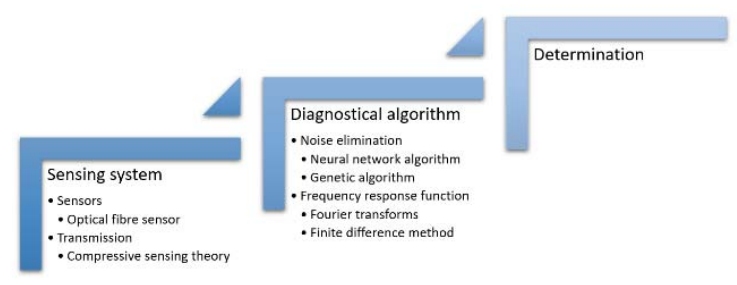

Fig. 3. Structural health monitoring process

\section{Conclusion}

The study of structural health monitoring in tall buildings is still lack of focus and further attention should be paid in this area. And the basic idea proposed in this paper is learned from the successful applications in bridges. A brief description of how these can be applied has been shown in Chengdu Greenland Center: The data like natural frequency are collected using optical fire sensors deployed along the core-tube. And neural network algorithm is used to eliminate the noise of the environment. Then the condition can be diagnosed by solving the frequency response function or impulse response function. However, owing to the lack of database, some examples are not demonstrated in a numerical way which can be improved in the future as the improvement of database in SHM of tall buildings. In conclusion, the SHM can be applied in tall buildings to diagnose the damages as it is introduced above.

Despite the quick development of structural health monitoring in skyscrapers, there are still lots of drawbacks of this application. First of all, the identification algorithms require a large amount of test results to form the database. And because of the short development time of SHM, this database is not adequate to support all kinds of tall buildings. Another huge challenge is that the natural frequency may not change noticeably owing to the small damages. This can be handled using more accurate sensors and some amplified algorithms.

\section{Acknowledgment}

First and foremost, I would like to show my deepest gratitude to my supervisor, Professor Oral Buyukozturk for his dedicated help. Without his perspective instruction and enlightening suggestions, I would not finish this paper. And I want to extend my thanks to Mr. Jiabin Chen for his help, who provided a lot of useful information and advice for me. 


\section{References}

1. J.M. Ko \& Y.Q. Ni. Technology developments in structural health monitoring of large-scale bridges. Engineering Structure, 14 July, 2005, pp. 1715-1725.

2. A. Miyamoto. Encyclopedia of Structural Health Monitoring. In: Usage Management of Civil Structures. Ube, Japan: Yamaguchi University, 2009.

3. C.R. Farrar, N.A. Lieven. Damage prognosis: the future of structural health monitoring. Philosophical Transactions of Royal Society A, December, 2007.

4. L. Hui, B. Yue-quan, L. Shun-long \& Z. Dong-yu. Data science and engineering for structural health monitoring. Engineering mechanics, August, 2015.

5. H. Chen. Structural health monitoring of large civil engineering structures. 1st edition ed. University of Greenwich, UK: John Wiley \& Sons, 2018.

6. Maia, et al. Theoretical and Experimental Modal Analysis. Taunton, Somerset, UK: Research Studies, 1997.

7. Y. Jin-cha, W. Choi. Deep learning-based Crack Damage detection using convolutional neural network. Computer-aided civil and infrastructure engineering, 2017, pp. 361-378.

8. C. Yu Research on Distributed Strain crack detection method based on stacked convolutional autoencoder, Xi an, China: s.n, 2019.

9. The Skyscraper Center, 2020. Chengdu Greenland

Tower. [Online] Available at:

http://www.skyscrapercenter.cn/building/chengdugreenland-tower/12845[Accessed 3 July 2020]. 